ARTí́culo

\title{
Reclutamiento del coral Acropora palmata sobre sustratos de dos materiales
}

Recruitment of the coral Acropora palmata on substrates of two materials

\section{Neidy P. Cetz-Navarro, ${ }^{1,2}$, Julio Espinoza-Avalos ${ }^{3 *}$, Alejandro Vega-Zepeda ${ }^{3}$, Ana I. Cerón-Flores ${ }^{4}$, Rodolfo Raigoza-Figueras ${ }^{4}$ y Eugenio de J. Carpizo-Ituarte ${ }^{5}$}

${ }^{1}$ Posgrado en Oceanografía Costera, Instituto de Investigaciones Oceanológicas-Facultad de Ciencias Marinas, Universidad Autónoma de Baja California, Ensenada, Baja California, México

${ }^{2}$ Universidad de Quintana Roo, División de Ciencias e Ingeniería, Boulevard Bahía s/n esq. Ignacio Comonfort, Del Bosque, Chetumal, Quintana Roo, 77010, México

${ }^{3}$ El Colegio de la Frontera Sur, Avenida Centenario km 5.5, Colonia Pacto Obrero Campesino, Chetumal 77014, Quintana Roo, México. *jespino@ecosur.mx

${ }^{4}$ Acuario XCARET, Playa del Carmen, Quintana Roo, México

${ }^{5}$ Instituto de Investigaciones Oceanológicas, Universidad Autónoma de Baja California, Ensenada, Baja California, México

Abstract.- During recruitment of corals, substrate characteristics influence the density and the spatial distribution of recruits. The influence of the material (limestone and sand) and of 4 groups arrangements (limestone/up, limestone/down, sand/up and sand/ down) in the distribution of the Acropora palmata recruits in 4 areas of the substrate used (upper face, side faces, bottom faces and base) was evaluated. The number of recruits per substrate was higher in limestone (median= 15.0) than in sand $(2.0)$. Regarding the areas of recruitment, the upper face in limestone and the upper and side faces in sand presented greater number of recruits than the other areas of the substrate; notably the upper faces received more light during the conditioning of the substrates. Concerning the groups, the number of recruits per substrate was higher in limestone groups (limestone/up $\approx$ limestone/down) than in sand groups (sand/up > sand/down). The relatively low number of A. palmata recruits obtained $\left(12500 \mathrm{~m}^{-2}\right)$ was, however, 3-4 orders of magnitude higher than the recruits obtained in Caribbean coral reefs (3-39 $\left.\mathrm{m}^{-2}\right)$, with an abundance dominated by opportunistic corals that do not provide the ecological qualities fulfilled by A. palmata. The implantation in the field of recruits obtained in aquarium remains as a viable alternative to recover populations of this critically endangered coral species, which substantially contributes to the structural complexity of Caribbean coral reefs.

Key words: Sexual reproduction, coral recruitment, aquarium coral culture, coral reefs, Mexican Caribbean

Resumen.- Durante el reclutamiento de los corales, las características de los sustratos influyen en la densidad y la distribución espacial de los reclutas. Se evaluó la influencia del material (conchuela y arena) y de 4 combinaciones denominadas grupos (conchuela/arriba, conchuela/abajo, arena/arriba y arena/abajo) en la distribución de los reclutas del coral Acropora palmata en las 4 áreas de los sustratos utilizados (cara superior, caras laterales, caras inferiores y base). En los materiales utilizados, el número de reclutas por sustrato fue mayor en la conchuela (mediana $=15,0)$ que en la arena $(2,0)$. En las áreas del sustrato, la cara superior en la conchuela y las caras superior y laterales en la arena tuvieron mayor número de reclutas que las otras áreas del sustrato; cabe señalar que las caras superiores fueron las que recibieron más luz durante el acondicionamiento de los sustratos. En los grupos, el número de reclutas por sustrato fue mayor en los grupos de conchuela (conchuela/arriba $\approx$ conchuela/ abajo) que en los de arena (arena/arriba > arena/abajo). El relativo bajo número de reclutas de $\mathrm{A}$. palmata que se obtuvo (12500 $\mathrm{m}^{-2}$ ) fue, sin embargo, 3-4 órdenes de magnitud mayor que la obtenida en arrecifes del Caribe (3-39 $\left.\mathrm{m}^{-2}\right)$, con una abundancia dominada por corales oportunistas que no proveen las cualidades ecológicas de A. palmata. La siembra en el campo de reclutas obtenidos en acuario permanece como una alternativa viable para recuperar las poblaciones de esta especie en peligro crítico de extinción, la cual contribuye sustancialmente en la complejidad estructural de arrecifes coralinos del Caribe.

Palabras clave: Reproducción sexual, reclutamiento de corales, cultivo de corales en acuario, arrecifes coralinos, Caribe mexicano 


\section{INTRODUCCIÓN}

La degradación de los arrecifes coralinos generalmente involucra la disminución de la cobertura de corales pétreos y el incremento de la cobertura de macroalgas (Hughes 1994), que pueden inhibir el asentamiento de larvas y la supervivencia de reclutas de corales (Birrell et al. 2005, Ritson-Williams et al. 2010, Webster et al. 2015). Los reclutas de corales observados en años recientes en el Caribe son principalmente de especies incubadoras oportunistas, de vida corta y relativamente pequeñas, y se han reportado mucho menos de especies liberadoras de gametos, de vida larga y gran complejidad estructural (Arnold \& Steneck 2011, Huntington et al. 2011), que proporcionan biodiversidad, funcionamiento del ecosistema y servicios ambientales asociados, como la protección costera (Alvarez-Filip et al. 2009). Bajo la perspectiva de la degradación de los arrecifes coralinos, una de las alternativas que se está implementando para intentar su recuperación es la reproducción sexual de corales en condiciones de acuario (Rinkevich 1995, Petersen et al. 2006, Ritson-Williams et al. 2010), a través de la cual se trata de mantener la diversidad genética de las poblaciones (Rinkevich 1995). A diferencia de lo anterior, la reproducción asexual de fragmentos de coral, como se realiza actualmente, reduce la diversidad genética de las poblaciones. Otra ventaja del cultivo ex situ de corales es potencialmente el éxito reproductivo de los corales observado en acuarios públicos (Petersen et al. 2007a), a diferencia del registrado in situ, donde la mortalidad de los reclutas puede alcanzar 50-88\% entre los primeros meses y antes de los 2 años (Ritson-Williams et al. 2010, Arnold \& Steneck 2011, Miller 2014). En el maricultivo de los corales pétreos (iniciando desde la fecundación) existen variantes en las condiciones para implementarlo (Rinkevich 1995, Szmant \& Miller 2006, RitsonWilliams et al. 2010); en las primeras etapas de desarrollo coralino es preponderante encontrar las condiciones de cultivo que aseguren la mayor viabilidad de los reclutas de una especie.

Con respecto al sustrato de reclutamiento, se ha observado que las larvas competentes pueden tener mayor afinidad para metamorfosearse en función del tipo de material, forma, textura y color, con diferente reclutamiento espacial en las distintas partes del sustrato. Estas preferencias dependen de las condiciones ambientales a las que se someten, así como la especie de coral involucrada (Petersen et al. 2005, 2007b; Szmant \& Miller 2006, Arnold \& Steneck 2011, Davies et al. 2013). Los materiales que se han utilizado para el reclutamiento coralino son carbonato de calcio, plástico, concreto, granito, acero, gabro, arenisca ('sandstone'), esqueleto de coral, terracota, madera, cerámica, caolinita y algas coralinas incrustantes (Petersen et al. 2005, Creed \& De Paula 2007, Burt et al. 2009, Ritson-Williams et al. 2010, Mason et al. 2011, Salinas-de-León et al. 2011). La preparación de los sustratos para reclutas requiere diferentes tiempos de elaboración y costos, y es recomendable encontrar un sustrato que sea de rápida elaboración, económico, en el que se adhiera el mayor número posible de reclutas y que pueda ser fácilmente incorporado en el fondo marino, si se desea implantarlo en el mar posteriormente. La distribución de los reclutas (i.e., cómo usan el espacio) en los sustratos depende de las especies (Szmant \& Miller 2006, Ritson-Williams et al. 2010); por ejemplo, Orbicella faveolata (= Montastraea faveolata (Ellis \& Solander, 1786)), sensu Budd et al. 2012) prefiere reclutarse en la parte inferior de los sustratos, mientras que Acropora palmata (Lamarck, 1816) se recluta en la parte superior de los mismos. Aunque A. palmata prefiere reclutarse en la superficie superior de sustratos de roca caliza, tejas de barro, escombros de arrecife (Szmant \& Miller 2006) y algas coralinas incrustantes (Ritson-Williams et al. 2010), durante el esfuerzo de repoblamiento de $A$. palmata llevado a cabo en el norte de Quintana Roo, México, se ha observado que en sustratos cilíndricos de arena cementada, los reclutas se fijan menos en la parte superior, por lo que para propiciar que se recluten en esa parte del sustrato, se colocan «boca abajo» o invertidos (A. Banaszak, obs. pers.). Estos resultados sugieren la necesidad de continuar ensayando el reclutamiento de A. palmata en otros sustratos de diferentes formas y materiales, que ayuden a obtener mayor densidad, desarrollo y supervivencia de los reclutas en condiciones de acuario.

El coral A. palmata es una especie hermafrodita que forma sus gametos asincrónicamente; es decir, primero se forman los gametos femeninos y posteriormente los masculinos. Ambos gametos son liberados en un evento anual en el verano (Szmant 1986). Este coral pétreo es una de las especies más importantes formadoras de estructura de arrecifes someros del Caribe (Miller et al. 2002), y actualmente está sujeta a protección especial en México (SEMARNAT 2010) ${ }^{1}$ y con categoría «En peligro crítico de extinción» por la Unión Internacional para la Conservación de la Naturaleza (IUCN; Aronson et al. 2008), debido a que sus poblaciones han sido muy diezmadas, principalmente por enfermedades (Aronson \& Pretch 2001, Sutherland et al. 2011) y huracanes (Hughes et al. 1987,

${ }^{1}$ SEMARNAT. 2010. Norma Oficial Mexicana NOM-059-SEMARNAT-2010, Protección ambiental- Especies nativas de México de flora y fauna silvestres-. Categorías de riesgo y especificaciones para su inclusión, exclusión o cambio. Lista de especies en riesgo. Diario Oficial de la Federación. México 30/12/2010, México, D.F., 78 pp. <http://dof.gob.mx/nota_detalle.php?codigo=5173091\&fecha=30/ $12 / 2010>$ 
Aronson \& Pretch 2001). En consecuencia, además de proteger a las poblaciones existentes, es necesario revertir la tendencia de degradación arrecifal a través de incrementar las colonias coralinas por medio de métodos de reproducción sexual (Garrison \& Ward 2012, Young et al. 2012). La información generada sobre los embriones, larvas y reclutas de A. palmata, obtenidos a través de la reproducción sexual (Szmant \& Miller 2006, Ritson-Williams et al. 2010, Mason et al. 2011, Randall \& Szmant 2009, Medina-Rosas et al. 2013), es relativamente reciente y sigue siendo relevante comparar diferentes formas y materiales de sustratos de reclutamiento para incrementar el éxito en la producción de reclutas en acuario y la posterior implantación de juveniles en el fondo marino.

El objetivo de este estudio fue evaluar la influencia del material (conchuela y arena cementada), en sustratos cúbicos con forma de $\mathrm{T}$, considerando además 4 combinaciones de material que se denominaron grupos (i.e., la combinación de material y posición: conchuela/arriba, conchuela/abajo, arena/ arriba y arena/abajo), en la distribución espacial de los reclutas de A. palmata. La distribución espacial de los reclutas se cuantificó en 4 áreas de cada sustrato (cara superior, caras laterales, caras inferiores y base). El número total y el mapeo de los reclutas (i.e., pólipos primarios) se obtuvo mediante la cuantificación en cada sustrato y en sus respectivas áreas. La hipótesis de trabajo fue que el reclutamiento de A. palmata sería diferente, de acuerdo al material, grupos y áreas de los sustratos. Adicionalmente, se estimó el porcentaje de pólipos (reclutas) primarios a partir de los embriones y las larvas iniciales, para valorar la eficiencia del sistema empleado para obtener los reclutas en los sustratos.

\section{Materiales Y MÉTODOS}

\section{Elaboración y aCONDICIONAMIENTo DE LOS SUSTRATOS}

Se elaboraron sustratos de 2 materiales. Los primeros, 168 sustratos de conchuela (roca sedimentaria con conchas de moluscos visibles), se elaboraron a partir de lozas para piso $(30 \times 15 \times 1,6 \mathrm{~cm})$ de acabado mate. Las lozas se cortaron con una sierra de disco para obtener cubos de $\sim 2$ × 2 × 1,6 $\mathrm{cm}$. Posteriormente, cada cubo se esculpió con un minitaladro Dremel 3000, hasta obtener una forma de T cúbica, con 4 áreas identificables: cara superior $\left[4 \mathrm{~cm}^{2}(2 \mathrm{~cm} \mathrm{x} 2 \mathrm{~cm})\right]$, caras laterales [5,6 $\mathrm{cm}^{2}(0,7 \mathrm{~cm} \times 2 \mathrm{~cm} \times 4$ lados $\left.)\right]$, caras inferiores y base (Fig. 1). Los sustratos del segundo material se elaboraron con arena blanca de playa. Primero, 56 de los sustratos de conchuela se separaron al azar para utilizarlos en la elaboración de un molde flexible de silicón francés, que se dejó secar por 3 h. Una mezcla de $50 \%$ arena y $50 \%$ de cemento blanco con agua de mar se vertió en el molde, y se dejó secar a temperatura ambiente por al menos $12 \mathrm{~h}$. El proceso se repitió en 3 ocasiones para obtener 168 sustratos de arena. La finalidad de la similitud en la forma de ambos sustratos fue que la respuesta del reclutamiento coralino de $A$. palmata fuera influenciada por el material, no por la forma.
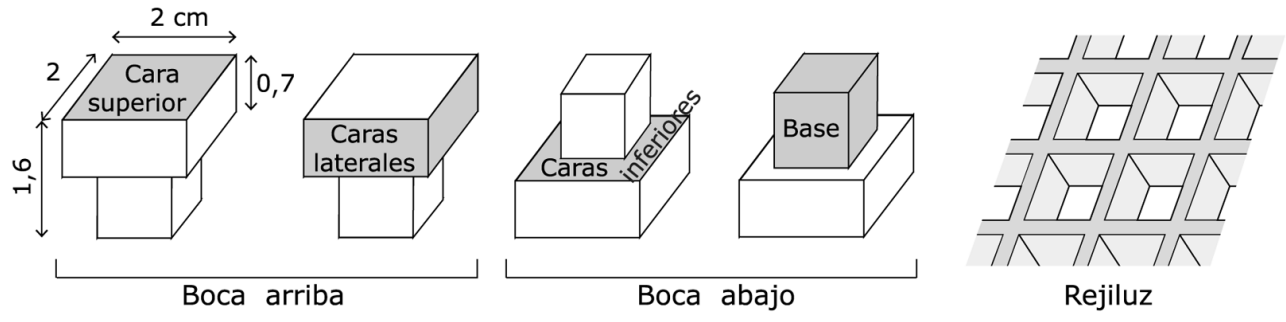

Figura 1. Sustratos en forma de T (de conchuela y de arena), los cuales se colocaron boca arriba y boca abajo sobre rejillas de poliestireno en cuadros (rejiluz). El reclutamiento en cada sustrato se analizó en 4 áreas (cara superior, caras laterales, caras inferiores y base), para cuantificar el número de reclutas por área y por cada sustrato / T-shaped substrates (made of limestone and sand), which were placed facing up and facing down on gridded polystyrene (rejiluz). Recruitment on each substrate was analyzed in 4 areas (upper face, side faces, bottom faces and base), to quantify the number of recruits per area and per each substrate 
El 12 de junio de 2015, dos meses antes de la liberación de los gametos de A. palmata, ambos sustratos se colocaron boca arriba en dos rejillas de poliestireno en cuadros (rejiluz, Fig. 1), una para cada tipo de sustrato. Las bases de los sustratos se acoplaron en los cuadros de la rejiluz, y se colocaron sin que sus caras laterales estuvieran en contacto, solo los vértices de las caras superiores. En esa posición se mantuvieron sumergidos en agua de mar en el Acuario del Parque Xcaret para su acondicionamiento. Durante ese tiempo ocurrió la formación de biopelículas, pero no la fijación de algas calcáreas incrustantes visibles a ojo u otros macroorganismos marinos.

\section{Fertilización}

Gametos provenientes de 10 colonias de A. palmata se recolectaron el 07 de agosto de 2015, una semana después de luna llena, en el arrecife de Punta Venado (20³1'57"N, $87^{\circ} 10^{\prime} 26^{\prime \prime O}$ ), ubicado a $8 \mathrm{~km}$ del Parque Xcaret, en Playa del Carmen, Quintana Roo, México. Los paquetes con gametos (masculinos + femeninos) se recolectaron a las 22:05 h, utilizando redes cónicas invertidas (con malla de luz de 100 $\mu \mathrm{m})$. La parte ancha de las redes, que contenía pequeños plomos, se colocó sobre las colonias; la parte angosta finalizaba con una boya y un frasco plástico invertido que mantenía la red a flote, para recolectar los gametos. A bordo de la lancha, a las 22:20 h, los paquetes de gametos de color rosáceo de todas las colonias se mezclaron en una nevera portátil, en aproximadamente $8 \mathrm{Lde}$ agua de mar filtrada (AMF). El AMF que se utilizó en este trabajo fue pasada a través de filtros de 20,10 y $5 \mu$ m e irradiada con luz UV. Los paquetes se agitaron suavemente con una pipeta plástica para romper las paredes y liberar a los gametos, lo que puede ocurrir después de $1 \mathrm{~h}$ (Szmant \& Miller 2006). La mezcla de gametos se trasladó al Parque Xcaret, donde después de 2 h (00:30 h del 08 de agosto) se dio por concluida la fertilización. Al concluir la fertilización, los cigotos que flotaban en la superficie del agua de mar se recolectaron con pipetas plásticas de transferencia y se depositaron en recipientes plásticos separadores de grasa, los cuales contenían alrededor de $1 \mathrm{Lde}$ AMF. En estos recipientes, el exceso de espermatozoides (que no participaron en la fertilización) se eliminaron mediante varios recambios de AMF (usando para desaguar la boquilla ubicada en la parte inferior del recipiente), hasta que el AMF quedó transparente.

\section{MANTENIMIENTO DE EMBRIONES Y LARVAS}

Al finalizar los recambios de agua, $12 \mathrm{~mL}$ de huevos/embriones se distribuyeron en 6 cubetas de $20 \mathrm{~L}$ ( $2 \mathrm{~mL}$ por cubeta), dentro de un tanque exterior ( 1 x 1 x $1 \mathrm{~m}$ ) ubicado en un área con techo de lámina de policarbonato transparente. Se estimó el número de huevos/embriones contenidos en $12 \mathrm{~mL}$, calculando primero el volumen de un embrión, considerándolo esférico y utilizando el diámetro de los huevos de A. palmata (300 $\mu \mathrm{m})$ reportado por Szmant (1986). Con la equivalencia entre $\mathrm{mm}^{3} \mathrm{y}$ mL, se calculó cuántos embriones ocuparían 12 mL ( 250000 huevos/embriones). Las cubetas de cultivo se construyeron previamente, como sigue: cada cubeta se cortó en dos partes y se traslaparon $5 \mathrm{~cm}$; entre ellas se colocó un fondo de red (con malla de luz de $100 \mu \mathrm{m}$ ). Ambas partes se pegaron con silicón y se aseguraron con cinchos, para evitar la separación de las partes y el desprendimiento de la red. En la periferia de la malla, dentro de la cubeta, se colocó silicón para evitar la pérdida de embriones y larvas. El cultivo de embriones/larvas se mantuvo con flujo continuo de AMF y a temperatura ambiente; la luz ambiental se disminuyó, colocando una capa de malla sombra sobre todo el techo. Después de 4 días, se observó que la mayoría de los embriones se había transformado en larvas (las cuales activamente nadaban y empezaron a reptar en el fondo de la malla de las cubetas); éstas y el resto de los embriones se trasladaron a las condiciones de reclutamiento.

\section{Reclutamiento}

Al cuarto día, todas las larvas y los pocos embriones que quedaban en las 6 cubetas se mezclaron en un recipiente con 10 L de AMF y se estimó el número total de embriones/larvas. El agua de mar se agitó suavemente, se extrajo $1 \mathrm{~mL}$ de agua y se contó el número de embriones/larvas; este procedimiento se repitió 5 veces, para obtener el número promedio de larvas por $\mathrm{mL}$ de agua, con lo que se calculó el número de embriones/ larvas ( 64000) en los 10 L de agua de mar. Luego, el agua se mezcló nuevamente y se dividió equitativamente en 12 cajas plásticas (833 mL, 5300 embriones/larvas, por caja), colocadas en otro tanque $(2 \times 1,5 \times 1 \mathrm{~m})$ en condiciones similares al cultivo de embriones y larvas. Las cajas ( $43 \mathrm{x} 28 \mathrm{x}$ $16 \mathrm{~cm}$ ) se construyeron previamente con paredes laterales de malla de luz de $175 \mu \mathrm{m}$ (ver Fig. 1 de Petersen et al. 2006), para permitir el flujo de agua. Para disminuir más la luz ambiental en las cajas, se colocó malla sombra sobre la parte superior y alrededor de las paredes del tanque de reclutamiento. Las cajas se asentaron sobre una mesa hecha de rejiluz (sostenida por soportes de pvc) elevada $\sim 0,8 \mathrm{~m}$ del fondo del tanque, quedando emergidas una mínima parte de las paredes de las cajas plásticas. El arreglo de las cajas permitió confinar a las larvas y observar con mayor detalle su actividad, sin manipularlas.

En el fondo de cada caja se colocó rejiluz y sobre ésta se colocaron los 4 grupos de sustratos (mezcla de 2 materiales y 2 posiciones). Así, en cada caja se colocaron intercaladamente 7 sustratos de conchuela boca arriba, 7 de conchuela boca abajo, 7 de arena boca arriba y 7 de arena boca abajo. En total, 336 sustratos se distribuyeron en las 12 cajas de 
reclutamiento. Las bases de los sustratos boca arriba se acoplaron a los cuadros de rejiluz, mientras que la cara superior de los colocados boca abajo quedó asentada sobre la rejiluz. Como en el acondicionamiento de los sustratos, las caras laterales de los diferentes grupos de sustratos no se tocaron.

Una semana después de trasladar las larvas a las cajas de reclutamiento, los sustratos se revisaron por la noche (iluminándolos con una lámpara fluorescente de luzazul NightSea Bluestar y un par de lentes con filtro amarillo), para observar, a simple vista y con la ayuda de un estereoscopio, si todas las larvas ya se habían asentado y metamorfoseado sobre los sustratos. Después de 14 días de haber depositado las larvas en las cajas de reclutamiento, se observó la ausencia de larvas y se incrementó la cantidad de reclutas. Entonces, con la ayuda de la lámpara fluorescente se cuantificó el número de pólipos primarios vivos que se reclutaron en cada material (conchuela y arena) y para los 4 grupos (conchuela/arriba, conchuela/abajo, arena/arriba y arena/abajo), en las 4 áreas de cada sustrato definidas inicialmente (Fig. 1). El número total de reclutas se obtuvo sumando los reclutas de las 4 áreas de cada sustrato.

Se estimó y comparó la densidad de reclutas registrados en el campo, transformando los valores a reclutas $\mathrm{m}^{-2}$. El área de los sustratos se estimó solo considerando la cara superior y las caras laterales $\left(=9,6 \mathrm{~cm}^{2}\right.$ en cada sustrato), ya que los reclutas de A. palmata sobre las caras inferiores y la base fueron mínimos y virtualmente inexistentes, respectivamente.

\section{AnÁlisis estadísticos}

Con la finalidad de evaluar el reclutamiento de A. palmata entre materiales, los datos del número de reclutas de $A$. palmata de los sustratos completos de conchuela y arena se analizaron con una prueba U de Mann-Whitney debido a que los datos no cumplieron los supuestos de normalidad (prueba de Kolmogorov-Smirnov, $P<0,05$ ) y homocedasticidad (prueba de Levene, $P<0,05)$. Para evaluar, por separado, el efecto de cada material de los sustratos, los datos del número de reclutas de A. palmata de la cara superior, las caras laterales, las caras inferiores y la base (i.e., cada sustrato desglosado en 4 áreas) se analizaron con ANDEVA de 1 vía no paramétricos (prueba de Kruskal-Wallis). Para evaluar el efecto de los 4 grupos (material/posición) de sustratos en el reclutamiento, los datos del número de reclutas por área y de los sustratos completos también se analizaron con pruebas de Kruskal-Wallis porque los datos no fueron normales (Kolmogorov-Smirnov, $P<0,05$ ) ni homocedásticos (Levene, $P<0,05$ ). Posteriormente, se utilizaron pruebas de Dunn, para la comparación de pares entre grupos.

\section{Resultados}

Sobre ambos sustratos se registraron 3693 pólipos primarios del coral A. palmata, los cuales representaron el 1,5\% del total de los embriones ( 250000) depositados en las cubetas, y el 5,8\% del total de las larvas ( 64000) depositadas en las cajas de reclutamiento.

\section{Reclutas de $A$. PALMATa SOBRe los MATERIAles DE SUSTRATO}

\section{Sustratos COMPletos}

Del total de los pólipos primarios registrados, 3000 se encontraron sobre los sustratos de conchuela (1742 boca arriba y 1258 boca abajo) y 693 sobre los de arena ( 542 boca arriba y 151 boca abajo). El número de reclutas de $A$. palmata fue diferente entre los sustratos de los materiales conchuela y arena (Mann-Whitney, $\mathrm{U}=5996,00, P=0,001$ ): en los sustratos de conchuela se encontró un mayor número de reclutas (mediana $(\mathrm{Me})=15,0)$ que en los sustratos de arena $(\mathrm{Me}=2,0)($ Fig. 2$)$.

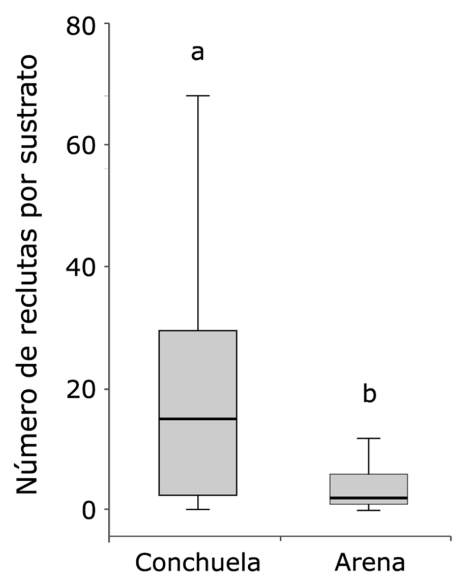

Figura 2. Mediana (línea horizontal gruesa), cuartiles $25-75 \%$ (caja gris) y valores mínimo y máximo de reclutas de Acropora palmata por sustrato en ambos materiales (conchuela vs. arena), sin importar la posición (boca arriba y boca abajo) en que se colocaron. Las letras sobre los diagramas de caja denotan la diferencia entre los materiales. Ver detalles en el texto / Median (thick horizontal line), 25-75\% quartiles (gray box) and minimum and maximum values of Acropora palmata recruits per substrate in both materials (limestone vs. sand), regardless the position (facing up and facing down) that they were placed. Letters on the boxplots denote the difference between materials. See details in the text 


\section{Áreas de LOS SUSTRATOS}

En los sustratos de conchuela, la cara superior (Fig. 3A) tuvo el mayor número de reclutas de A. palmata que el resto de las áreas; a su vez, las caras laterales tuvieron más reclutas que las caras inferiores y la base de los sustratos (Kruskal-Wallis, H= 319,70 , g.l. $=3, P=0,001$ ) (Figs. 3C y 4A). En los sustratos de arena, la cara superior (Fig. 3B) y las caras laterales tuvieron mayor número de reclutas que las caras inferiores y la base $(\mathrm{H}=126,58$, g.l. $=3, P=0,001)$ (Fig. 4B).

Reclutas de A. palmata sobre los grupos de SUSTRATOS

\section{Sustratos COMPLETOS}

Las larvas de A. palmata prefirieron metamorfosearse sobre los sustratos de conchuela, que sobre los de arena, independientemente si se encontraban boca arriba o boca abajo (H=71,55, g.l.= 3, $P=0,001)$ (Figs. 3A, B y 5). Los sustratos de arena presentaron diferencias en las dos posiciones, ya que los sustratos boca arriba tuvieron más reclutas que los sustratos boca abajo (prueba de Dunn, $P<0,05$ ) (Fig. 5).
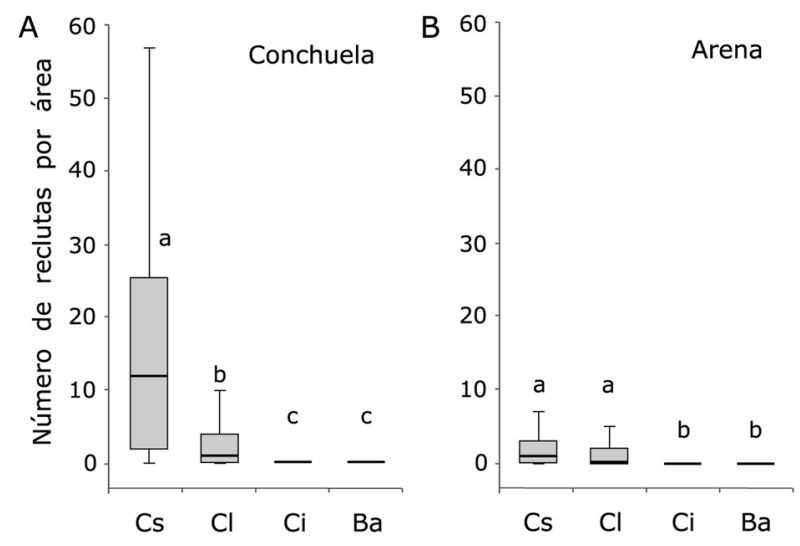

Figura 4. Mediana (línea horizontal gruesa), cuartiles 25-75\% (caja gris) y valores mínimo y máximo de reclutas de Acropora palmata por área [cara superior (Cs), caras laterales (Cl), caras inferiores (Ci) y base $(\mathrm{Ba})]$ de los sustratos en cada material, sin importar la posición (boca arriba y boca abajo) en que se colocaron. A) Reclutas por área en los sustratos de conchuela. B) Reclutas por área en los sustratos de arena. Las letras sobre los diagramas de caja denotan las diferencias entre lasáreas de cada material. Ver detalles en el texto y en la Fig. 1 / Median (thick horizontal line), $25-75 \%$ quartiles (gray box) and minimum and maximum values of Acropora palmata recruits per area [upper face (Cs), side faces (Cl), bottom faces (Ci) and base (Ba)] in the substrates of each material, regardless the position (facing up and facing down) that they were placed. A) Recruits per area in the limestone substrates. B) Recruits per area in the sand substrates. Letters on the boxplots denote the differences among the areas of each material. See details in the text and Fig. 1

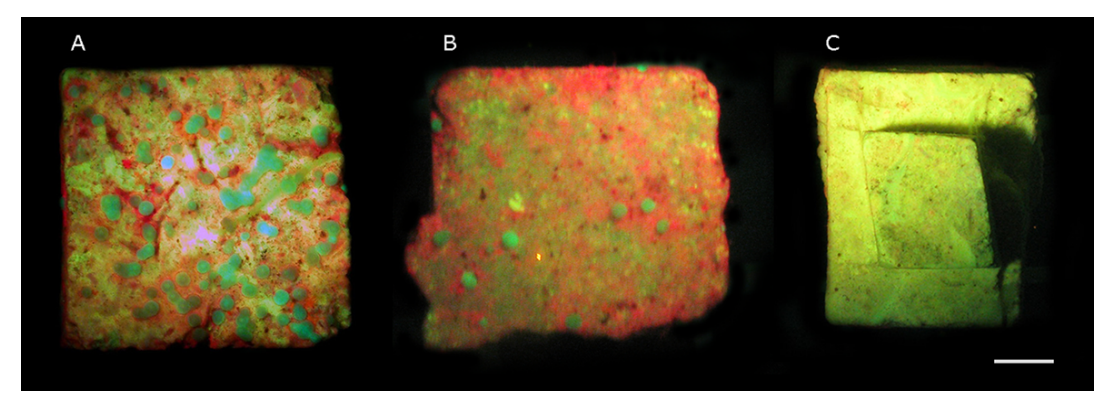

Figura 3. Sustratos con y sin pólipos primarios de Acropora palmata. A) Reclutas de A. palmata sobre la cara superior de un sustrato de conchuela boca arriba, B) reclutas de coral sobre la cara superior de un sustrato de arena boca arriba, y C) ausencia de reclutas de A. palmata en un sustrato de conchuela boca abajo. Los reclutas (puntos de color verde fluorescente) se iluminaron con una lámpara fluorescente de luz azul. Escala: $\mathbf{0 , 5} \mathrm{cm}$. Fotos: NP Cetz-Navarro / Substrates with and without primary polyps of Acropora palmata. A) A. palmata recruits on the upper face of a limestone substrate facing up, B) coral recruits on the upper face of a sand substrate facing up, and $C$ ) absence of $A$. palmata recruits on a limestone substrate facing down. Recruits (fluorescent green dots) were illuminated with a fluorescent lamp of blue light. Scale: $0.5 \mathrm{~cm}$. Photos: NP Cetz-Navarro 


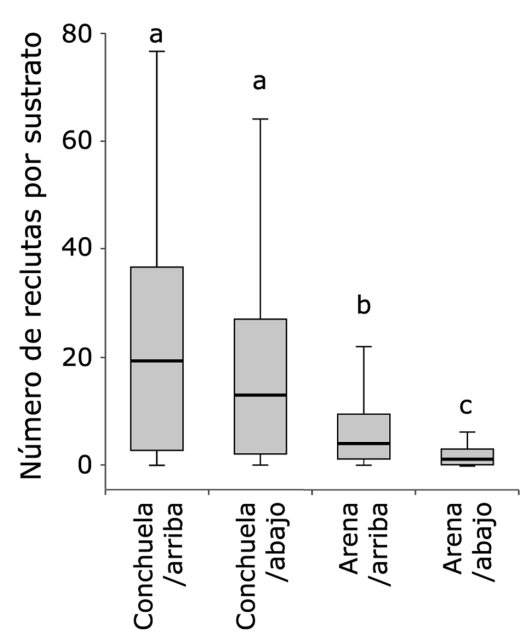

Figura 5. Mediana (línea horizontal gruesa), cuartiles $25-75 \%$ (caja gris) y valores mínimo y máximo de reclutas de Acropora palmata por sustrato en los 4 grupos evaluados [conchuela boca arriba (Conchuela/ arriba), conchuela boca abajo (Conchuela/ abajo), arena boca arriba (Arena/ arriba) y arena boca abajo (Arena/ abajo)]. Las letras sobre los diagramas de caja denotan las diferencias entre los grupos de los sustratos / Median (thick horizontal line), $25-75 \%$ quartiles (gray box) and minimum and maximum values of Acropora palmata recruits per substrate in the 4 evaluated groups [limestone facing up (Conchuela/arriba), limestone facing down (Conchuela/abajo), sand facing up (Arena/arriba) and sand facing down (Arena/abajo)]. Letters on the boxplots denote the differences among the groups of the substrates

\section{Áreas de los SUSTRATOS}

En la cara superior, el número de reclutas en los sustratos de conchuela fue similar en ambas posiciones y, a su vez, mayor que en los sustratos de arena; sin embargo, los sustratos de arena boca arriba presentaron más reclutas que en los sustratos de arena boca abajo $(\mathrm{H}=96,56$, g.l. $=3, P=0,001$; prueba de Dunn, $P<0,05)$ (Figs. 2A, 2B y 6). En las caras laterales, el número de pólipos primarios fue menor en los sustratos de arena boca abajo que en los sustratos de conchuela en ambas posiciones y arena boca arriba $(\mathrm{H}=23,92$, g.l. $=3, P=0,001$; prueba de Dunn, $P<0,05$ ) (Fig. 6). En las caras inferiores, los sustratos de conchuela boca arriba tuvieron menos reclutas que los sustratos de arena boca abajo $(\mathrm{H}=30,36$, g.l $=3, P=0,001$; prueba de Dunn, $P<0,05$ ) (Fig. 6). En la base, el número de reclutas de $A$. palmata fue similar entre todos los grupos $(\mathrm{H}=$ 17,67, g.l.= 3, $P>0,05)$ (Figs. 2C y 6 ).

\section{DisCuSIón}

Los resultados de este estudio demuestran que el número de reclutas de $A$. palmata fue diferente entre los materiales, los grupos y las áreas de los sustratos, de acuerdo con la hipótesis de trabajo. El material de los sustratos influyó en un reclutamiento diferencial de las larvas de A. palmata (7 veces mayor sobre conchuela que sobre arena), de manera similar a otros estudios que refieren, por ejemplo, que la densidad de reclutas de Tubastraea tagusensis fue diferente de acuerdo con el material (mayor en cemento que en cerámica y metal) de los sustratos (Creed \& De Paula 2007). Análogamente, Szmant \& Miller

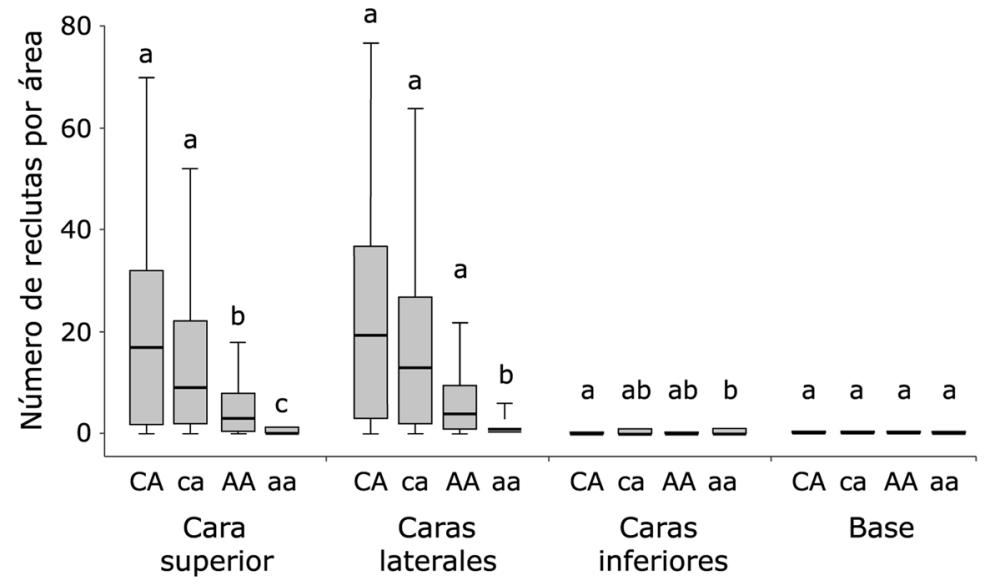

Figura 6. Mediana (línea horizontal gruesa), cuartiles 25-75\% (caja gris) y valores mínimo y máximo de reclutas de Acropora palmata por área en los 4 grupos evaluados [conchuela boca arriba (CA), conchuela boca abajo (ca), arena boca arriba (AA) y arena boca abajo (aa)]. Las letras sobre los diagramas de caja denotan las diferencias entre las áreas de los grupos / Median (thick horizontal line), 25-75\% quartiles (gray box) and minimum and maximum values of Acropora palmata recruits per area in the 4 evaluated groups [limestone facing up (CA), limestone facing down (ca), sand facing up (AA) and sand facing down (aa)]. Letters on the boxplots denote the differences among areas of the groups 
(2006) mostraron en el laboratorio que las larvas de Orbicella faveolata prefirieron reclutarse sobre sustratos de conchuela y yeso que sobre algas coralinas incrustantes, de manera similar a lo que ocurrió en otra investigación realizada en el campo (van Woesik et al. 2014). Aunque en los sustratos de conchuela hubo un mayor reclutamiento de A. palmata que en los de arena cementada, éstos son más fácil de elaborar y no se consume mucho tiempo para prepararlos, por lo que su uso es más económico.

Con respecto a las áreas y los grupos de los sustratos, el mayor reclutamiento registrado en la cara superior de sustratos de conchuela, independientemente si fueron colocados boca arriba o boca abajo, fue igual a lo registrado por Szmant \& Miller (2006) para larvas de A. palmata que se reclutaron sobre sustratos de caliza ( $\sin$ algas coralinas incrustadas) que fueron acondicionados ('curados') boca arriba, como ocurrió en este estudio. En contraste, el reclutamiento sobre la cara superior de sustratos de arena cementada sí fue diferente dependiendo de la posición que se colocaron; el reclutamiento fue mayor en los colocados boca arriba que los colocados boca abajo, lo cual evidencia que la naturaleza de los materiales de los sustratos imposibilita establecer como regla que las larvas de A. palmata se reclutan en la parte superior de sustratos, independientemente de su posición. Estos resultados enfatizan el valor de ensayar con diferentes materiales y posiciones de sustratos, y encontrar lo más adecuado para el reclutamiento de corales en condiciones de acuario. Cabe destacar que el área donde ocurrió el mayor reclutamiento de A. palmata (cara superior) de los sustratos, fue la que recibió más luz solar durante su acondicionamiento, lo que pudo favorecer la formación de biopelículas adecuadas que estimularon el asentamiento y metamorfosis de larvas competentes de A. palmata.

La discrepancia en la estimación de un número alto de embriones obtenidos y de larvas depositadas en las cajas de reclutamiento, pero un muy bajo número de pólipos primarios de A. palmata registrados en los sustratos, tienen dos posibles orígenes. Primero, tener muchos embriones en las cubetas pudo ser perjudicial, ya que aumentaron tanto los compuestos grasos que se liberaron durante el cultivo como la materia orgánica en descomposición de embriones muertos. Luego, el número de larvas depositadas ( 190 por sustrato en promedio) en las cajas de reclutamiento fue tal vez excesivo, ya que de ellas sólo se obtuvo $6 \%$ de reclutas ( 12 por sustrato en promedio). Así, la tasa de mortalidad de las larvas incrementa al aumentar su densidad (Vermeij et al. 2009), mientras que la mortalidad de las larvas al competir por el espacio de asentamiento sobre los sustratos no puede descartarse. Segundo, el número de reclutas registrados en este estudio se subestimó, ya que no se cuantificaron los reclutas que también se fijaron sobre la rejiluz y las paredes (incluyendo la malla) y el fondo de las cajas de reclutamiento, ni otros pólipos primarios calcificados que se mantuvieron flotando. Se ha sugerido que estos pólipos no se fijan debido, entre otras posibles causas, a cualidades insuficientemente propias de los sustratos (Harrison \& Wallace 1990, Sneed et al. 2015). Las causas (no mutuamente excluyentes) de la discrepancia entre muchas larvas y pocos reclutas indican que el protocolo seguido en acuario para el maricultivo de A. palmata requiere de ajustes que contribuyan al incremento en la obtención de reclutas sobre los sustratos experimentales. Cabe destacar que diversos organismos marinos bentónicos muestran mortalidades elevadas $(\leq 98 \%)$ en etapas tempranas (Rumrill 1990, Gosselin \& Qian 1997, Hunt \& Scheibling 1997) debido a diversos factores comola ausencia de un sustrato inadecuado (Rumrill 1990), el retraso de la metamorfosis, el estrés fisiológico y la competencia (Hunt \& Scheibling 1997).

El relativo bajo reclutamiento de $A$. palmata que se obtuvo en acuario, sin embargo, es muy superior al número de reclutas (ejemplares de uno a pocos pólipos, de 2 a 40 mm de diámetro; Edmunds 2000) que se obtiene en el campo (Raymundo \& Maypa 2004). Por ejemplo, en este estudio, la densidad promedio de reclutas en los sustratos $\left(12500 \mathrm{~m}^{-2}\right)$ fue 3-4 órdenes de magnitud mayor que la obtenida en arrecifes del Caribe por Edmunds (2000; $32 \mathrm{~m}^{-2}$ ), Ruiz-Zárate et al. (2003; $3 \mathrm{~m}^{-2}$ ), Moulding (2005; 6-39 $\mathrm{m}^{-2}$ ) y Lozano-Cortes \& Zapata (2015; $\left.6 \mathrm{~m}^{-2}\right)$. Los reclutas dominantes en los sustratos colocados en el campo pertenecieron a géneros como Agaricia, Porites y Siderastrea (van Woesik et al. 2014), corales oportunistas de tamaño pequeño, que disminuyen la complejidad estructural y biótica de los arrecifes del Caribe (Alvarez-Filip et al. 2013), todo lo opuesto a las cualidades ecológicas positivas que proveen las poblaciones de $A$. palmata. Por otra parte, el reclutamiento de $A$. palmata en el campo es nulo, raro o virtualmente inexistente (Porter \& Meier 1992, Quinn \& Kojis 2005, Williams et al. 2008), por lo que se ha sugerido que la recuperación de las poblaciones por reproducción sexual puede ser fallida (Williams et al. 2008) o que las poblaciones estén destinadas a la extinción (Japaud et al. 2015). Es decir, el maricultivo de A. palmata, donde es posible obtener miles de reclutas que luego pueden implantarse en el campo, sigue siendo una alternativa potencial muy valiosa para buscar la recuperación de las poblaciones de A. palmata. Lo anterior demuestra lo prometedor que resulta producir corales, utilizando métodos de reproducción sexual en condiciones de laboratorio, como estrategia fundamental para desarrollar programas de repoblamiento y recuperación de arrecifes. 
En conclusión, las larvas de A. palmata se reclutaron de manera diferencial, de acuerdo al material de los sustratos, áreas y grupos de sustratos. El reclutamiento fue mayor en los sustratos de conchuela que en los de arena, aunque la elaboración de los de conchuela fue más costosa. El mayor reclutamiento ocurrió en las caras superiores, las cuales recibieron mayor cantidad de luz durante el acondicionamiento de los sustratos. La cantidad de reclutas obtenidos en los sustratos fue muy baja, al compararlos con el número de larvas existentes antes del reclutamiento, debido a que se subestimaron (al no contabilizar reclutas en las otras superficies del cultivo) y probablemente a la mortalidad de larvas al momento de metamorfosearse, lo que indica la necesidad de mejorar el protocolo en el cultivo de fases tempranas de A. palmata. Sin embargo, la densidad de reclutas obtenidos fue 3-4 órdenes de magnitud mayor a lo que se obtiene en el campo, lo cual alienta a seguir mejorando la obtención de reclutas en maricultivo. Estos reclutas pueden implantarse en el campo como una alternativa para recuperar las poblaciones de esta especie que se encuentra en peligro crítico de extinción, y cuya presencia es esencial en la formación de estructura tridimensional en los arrecifes coralinos del Caribe.

\section{Agradecimientos}

Agradecemos al personal del Acuario de Xcaret, especialmente a A Coronel, por todo su apoyo y ayuda durante todoel estudio; a los estudiantes VM Ayala-Montañez, VM Valenzuela-López, FG Llamas-Valenzuela, D Reyes-Cortés y J Calle-Treviño, por su ayuda en el trabajo de laboratorio; a JA Cohuó-Collí, por su ayuda en una parte del trabajo de campo; al personal de la UNAM y SECORE Foundation, por su contribución en el trabajo de campo; a JE Marrufo-Romero, EM Cetz-Navarro y G Le Cossec, por su ayuda en la elaboración de sustratos; a A Banaszak (UNAM) por proporcionar información a través de comunicación personal; y a A Díaz-de-León y OE DelgadoGonzález por su ayuda logística durante la estancia posdoctoral. Este trabajo fue financiado en parte por el CONACYT [Proyecto 257855 (JEA)], ECOSUR [a través de fondos federales (JEA)], el Acuario del Parque Xcaret (AICF y RRF) y recursos personales (NPCN). NPCN agradece el apoyo otorgado por el CONACYT (beca 290917-UABC) para realizar una estancia posdoctoral (en 2015) en la Universidad Autónoma de Baja California. Este trabajo fue parte de la UMA 'Parque Ecoarqueológico XCARET (INE/CITES/DFSYFSZOO-P0011-00-Q.ROO)', permiso autorizado por la SEMARNAT. Agradecemos particularmente las observaciones de un evaluador anónimo, que permitieron mejorar la versión final del manuscrito.

\section{LITERATURA CITADA}

Alvarez-Filip L, NK Dulvy, JA Gill, IM Côté \& AR Watkinson. 2009. Flattening of Caribbean coral reefs: region-wide declines in architectural complexity. Proceedings of the Royal Society B 276: 3019-3025.

Alvarez-Filip L, JP Carricart-Ganivet, G Horta-Puga \& R Iglesias-Prieto. 2013. Shifts in coral-assemblage composition do not ensure persistence of reef functionality. Scientific Reports 3: 3486.

Arnold SN \& RS Steneck. 2011. Settling into an increasingly hostile world: The rapidly closing "recruitment window" for corals. PLoS ONE 6(12): e28681. <doi:10.1371/ journal.pone.0028681>

Aronson RB \& WF Precht. 2001. White-band disease and the changing face of Caribbean coral reefs. Hydrobiologia 460: 25-38.

Aronson R, A Bruckner, J Moore, B Precht \& E Weil. 2008. Acropora palmata. The IUCN Red List of Threatened Species 2008: e.T133006A3536699. <http:// dx.doi.org/ $10.2305 /$ IU CN.UK.2008.RLTS. T133006A3536699.en.>

Birrell CL, LJ McCook \& BL Willis. 2005. Effects of algal turfs and sediment on coral settlement. Marine Pollution Bulletin 51:408-414.

Budd AF, H Fukami, ND Smith \& N Knowlton. 2012. Taxonomic classification of the reef coral family Mussidae (Cnidaria: Anthozoa: Scleractinia). Zoological Journal of the Linnean Society 166: 465-529.

Burt J, A Bartholomew, A Bauman, A Saif \& PF Sale. 2009. Coral recruitment and early benthic community development on several materials used in the construction of artificial reefs and breakwaters. Journal of Experimental Marine Biology and Ecology 373: 72-78.

Creed JC \& AF De Paula. 2007. Substratum preference during recruitment of two invasive alien corals onto shallow-subtidal tropical rocky shores. Marine Ecology Progress Series 330: 101-111.

Davies SW, MV Matz \& PD Vize. 2013. Ecological complexity of coral recruitment processes: Effects of invertebrate herbivores on coral recruitment and growth depends upon substratum properties and coral species. PLoS ONE 8(9): e72830. <doi:10.1371/journal.pone.0072830>

Edmunds PJ. 2000. Patterns in the distribution of juvenile corals and coral reef community structure in St. John, US Virgin Island. Marine Ecology Progress Series 202: 113-124.

Garrison VH \& G Ward. 2012. Transplantation of stormgenerated coral fragments to enhance Caribbean coral reefs: A successful method but not a solution. Revista de Biología Tropical 60: 59-70.

Gosselin LA \& PY Qian. 1997. Juvenile mortality in benthic marine invertebrates. Marine Ecology Progress Series 146: 265-282. 
Harrison PL \& CC Wallace. 1990. Reproduction, dispersal and recruitment of scleractinian corals. In: Dubinsky Z (ed). Coral reefs. Ecosystems of the world 25: 133-207. Elsevier, New York.

Hughes TP. 1994. Catastrophes, phase shifts, and large-scale degradation of a Caribbean coral reef. Science 265: 15471551.

Hughes TP, DC Reed \& MJ Boyle. 1987. Herbivory on coral reefs: community structure following mass mortalities of sea urchins. Journal of Experimental Marine Biology and Ecology 113: 39-59.

Hunt HL \& RE Scheibling. 1997. Role or early postsettlement mortality in recruitment of benthic marine invertebrates. Marine Ecology Progress Series 155: 269-301.

Huntington BE, M Karnauskas \& D Lirman 2011. Corals fail to recover at a Caribbean marine reserve despite ten years of reserve designation. Coral Reefs 30: 1077-1085.

Japaud A, C Bouchon, JL Manceau \& C Fauvelot. 2015. High clonality in Acropora palmata and Acropora cervicornis populations of Guadeloupe, French Lesser Antilles. Marine and Freshwater Research 66: 847-851.

Lozano-Cortés DF \& FA Zapata. 2015. Abundance and composition of juvenile scleractinian corals on a fringing reef (Little Reef) off San Andres Island, Colombian Caribbean. Marine Biology Research 11: 304-312.

Mason B, M Beard \& MW Miller. 2011. Coral larvae settle at a higher frequency on red surfaces. Coral Reefs 30: 667676.

Medina-Rosas P, AM Szmant \& RF Whitehead. 2013. $\mathrm{CO}_{2}$ enrichment and reduced seawater $\mathrm{pH}$ had no effect on the embryonic development of Acropora palmata (Anthozoa, Scleractinia). Invertebrate Reproduction \& Development 57: 132-141.

Miller MW. 2014. Post-settlement survivorship in two Caribbean broadcasting corals. Coral Reefs 33: 1041-1046.

Miller MW, AS Bourque \& JA Bohnsack. 2002. An analysis of the loss of acroporid corals at Looe Key, Florida, USA: 1983-2000. Coral Reefs 21: 179-182.

Moulding AL. 2005. Coral recruitment patterns in the Florida Keys. Revista de Biología Tropical 53: 75-82.

Petersen D, M Laterveer \& H Schuhmacher. 2005. Innovative substrate tiles to spatially control larvae settlement in coral culture. Marine Biology 146: 937-942.

Petersen D, ML Laterveer, D Van Bergen, M Hatta, $R$ Hebbinghaus, M Janse, R Jones, U Richter, T Ziegler, G Visser \& H Schuhmacher. 2006. The application of sexual coral recruits for the sustainable management of $e x$ situ populations in public aquariums to promote coral reef conservation-SECORE Project. Aquatic Conservation: Marine and Freshwater Ecosystems 16: 167-179.
Petersen D, J Falcato, P Gilles \& R Jones. 2007a. Sexual coral reproduction in live coral exhibits-current status and future perspectives. International Zoo Yearbook 41: 122-137.

Petersen D, M Laterveer \& G Visser. 2007b. Sexual recruitment of the corals Favia fragum and Agaricia humulis in a $30-\mathrm{m}^{3}$ exhibit aquarium: species-specific limitations and implications on reproductive ecology. Zoo Biology 26: 75-91.

Porter JW \& OW Meier. 1992. Quantification of loss and change in Floridian reef coral populations. The American Zoologist 32: 625-640.

Quinn NJ \& BL Kojis. 2005. Patterns of sexual recruitment of acroporid coral populations on the West Fore Reef at Discovery Bay, Jamaica. Revista de Biología Tropical 53: 83-89.

Randall CJ \& AM Szmant. 2009. Elevated temperature affects development, survivorship, and settlement of the elkhorn coral, Acropora palmata (Lamarck 1816). The Biological Bulletin 217:269-282.

Raymundo LJ \& AP Maypa. 2004. Getting bigger faster: Mediation of size-specific mortality via fusion in juvenile coral transplants. Ecological Applications 14: 281-295.

Rinkevich B. 1995. Restoration strategies for coral reefs damages by recreational activities: the use of sexual and asexual recruits. Restoration Ecology 3: 241-251.

Ritson-Williams R, VJ Paul, S Arnold \& RS Steneck. 2010. Larval settlement preferences and post-settlement survival of the threatened Caribbean corals Acropora palmata and A. cervicornis. Coral Reefs 29: 71-81.

Ruiz-Zárate MA, RC Hernández-Landa, C González-Salas, E Nuñez-Lara \& E Arias-González. 2003. Condition of coral reef ecosystems in central-southern Quintana Roo, Mexico (Part 1: stony corals and algae). Atoll Research Bulletin 496: 318-337.

Rumrill SS. 1990. Natural mortality of marine invertebrate larvae. Ophelia 32: 163-198.

Salinas-de-León P, A Costales-Carrera, S Zeljkovic, DJ Smith \& JJ Bell. 2011. Scleractinian settlement patterns to natural cleared reef substrata and artificial settlement panels on an Indonesian coral reef. Estuarine, Coastal and Shelf Science 93: 80-85.

Sneed JM, KH Sharp, KB Ritchie \& VJ Paul. 2015. The chemical cue tetrabromopyrrole from a biofilm bacterium induces settlement of multiple Caribbean corals. Proceedings of the Royal Society B-Biological Sciences 281. <doi:10.1098/rspb.2013.3086>

Sutherland KP, S Shaban, JL Joyner, JW Porter \& EK Lipp. 2011. Human pathogen shown to cause disease in the threatened eklhorn coral Acropora palmata. PLoS ONE 6(8): e23468. <doi:10.1371/journal.pone.0023468> 
Szmant AM. 1986. Reproductive ecology of Caribbean reef corals. Coral Reefs 5: 43-54.

Szmant AM \& MW Miller. 2006. Settlement preferences and post-settlement mortality of laboratory cultured and settled larvae of the Caribbean hermatypic corals Montastraea faveolata and Acropora palmata in the Florida Keys, USA. Proceedings of the 10th International Coral Reef Symposium 2: 43-49.

Van Woesik R, WJ Scott \& RB Aronson. 2014. Lost opportunities: Coral recruitment does not translate to reef recovery in the Florida Keys. Marine Pollution Bulletin 88: 110-117.

Vermeij MJA, JE Smith, CM Smith, RV Thurber \& SA Sandin. 2009. Survival and settlement success of coral planulae: independent and synergistic effects of macroalgae and microbes. Oecologia159:325-336.
Webster FJ, RC Babcock, M Van Keulen \& NR Loneragan. 2015. Macroalgae inhibits larval settlement and increases recruit mortality at Ningaloo Reef, Western Australia. PLoS ONE 10: e0124162. <doi:10.1371/ journal.pone.0124162>

Williams DE, MW Miller \& KL Kramer. 2008. Recruitment failure in Florida Keys Acropora palmata, a threatened Caribbean coral. Coral Reefs 27: 697-705.

Young CN, SA Schopmeyer \& D Lirman. 2012. A review of reef restoration and coral propagation using the threatened genus Acropora in the Caribbean and western Atlantic. Bulletin of Marine Science 88: 1075-1098.

Recibido el 5 de abril de 2016 y aceptado el 11 de octubre de 2016

Editor: Claudia Bustos D. 